\title{
HISTERECTOMIAS: ESTUDO RETROSPECTIVO DE 554 CASOS
}

\section{HYSTERECTOMIES: A RETROSPECTIVE STUDY OF 554 CASES}

\author{
Eddie Fernando Cândido Murta,ACBC-MG' \\ Juliana Delfino dos Reis ${ }^{2}$ \\ Juliana Misson Abrão \\ Juliana Muniz Miziara²
}

\begin{abstract}
RESUMO: Objetivo: A histerectomia é uma operação muito realizada, entretanto há poucos trabalhos na literatura nacional sobre suas indicações, técnica e complicações. O objetivo deste trabalho é avaliar estes procedimentos realizados na Disciplina de Ginecologia e Obstetrícia da Faculdade de Medicina do Triângulo Mineiro. Método: Estudo retrospectivo de 470 histerectomias abdominais e 84 vaginais foi conduzido analisando as indicações, tempo de cirurgia e internação, tipo de incisão e morbidez. Resultados: As principais indicações foram o mioma uterino e o prolapso uterino para as histerectomias abdominais e vaginais, respectivamente. As complicações intra-operatórias aconteceram em 3,4\% e as pós-operatórias em 2,4\% do total de casos. Nenhuma diferença estatística foi encontrada no número de complicações em relação ao tipo de incisão (vertical ou transversal). O tempo de cirurgia e o de hospitalização foram estatisticamente maiores nas incisões verticais. A hemorragia foi a mais freqüente complicação intra-operatória e a infecção da incisão operatória foi a mais freqüente no pós-operatório. Conclusões: A histerectomia é um procedimento de baixo risco, no entanto, a realização de revisões sobre indicações e complicações, e a pesquisa de melhores técnicas cirúrgicas são necessárias para torná-la cada vez mais segura.
\end{abstract}

Descritores: Histerectomia abdominal; Histerectomia vaginal; Indicações; Complicações; Incisão abdominal.

\section{INTRODUÇÃO}

A história da histerectomia remonta ao século XVI. A primeira operação deste tipo é creditada a Berengarius, que, em 1507, na cidade de Bolonha, realizou a retirada do útero através da vagina. Entretanto, há dúvidas sobre se a retirada foi total ou parcial. Não obstante, em 1560, é creditada a Andreas a realização da primeira ressecção uterina por via vaginal na cidade de Cruce. Após esta data, os dados sobre este procedimento não são claros. A maioria dos casos relatados é de extirpação parcial ou total do útero por inversão do órgão após parto. Somente no século XIX a histerectomia vaginal foi realizada com planejamento. As indicações, naquela época, eram para os casos de prolapso, retroversão aguda ou crônica e no câncer do colo uterino ${ }^{1}$.

A primeira exérese do útero pela via abdominal foi realizada por Heath em Manchester, Inglaterra, no ano de 1842, após quase 300 anos da primeira cirurgia vaginal. Ao que parece, a indicação da cirurgia foi por aumento de ová- rio; entretanto, durante a laparotomia foi encontrada uma massa sólida compatível com mioma e, então, realizou-se uma retirada uterina subtotal. No entanto, a primeira ressecção total por mioma com planejamento pré-operatório foi realizada por John Bellinger em 1846 nos Estados Unidos da América do Norte ${ }^{1}$.

Atualmente, esta operação é a mais praticada nos Estados Unidos, com cerca de 800.000 cirurgias/ano ${ }^{2,3}$. As principais indicações são ${ }^{3,4}: 1$ - Doença uterina benigna e/ ou sintomas: a) sangramento anormal, b) dor, sangramento ou aumento de volume do útero, c) prolapso, d) mioma, e) abortamento séptico e infecção puerperal, f) hemorragias obstétricas; 2 - Doenças benignas da tuba uterina e ovários: a) doença inflamatória pélvica, b) endometriose pélvica, c) gravidez ectópica; 3 - Neoplasias benignas e malignas (colo e corpo uterino, tuba, ovário, órgãos adjacentes); 4 - Doença trofoblástica gestacional; 5 - Indicações incomuns: a) problemas cervicais: estenose cervical, b) malformações, c) dor pélvica crônica, d) síndrome da pelve congesta, e) esterilização.

1. Professor Adjunto da Disciplina de Ginecologia e Obstetrícia.

2. Acadêmica de Medicina.

Recebido em 22/2/2000

Aceito para publicação em 13/7/2000

Trabalho realizado na Disciplina de Ginecologia e Obstetrícia da Faculdade de Medicina do Triângulo Mineiro. 
A técnica deste procedimento é consagrada com poucas variações entre os serviços ${ }^{4,5}$. O número de complicações é pequeno e a morbidez é baixa ${ }^{5}$.

Apesar de ser uma operação muito realizada, há poucos trabalhos na literatura nacional sobre suas indicações, técnica e complicações. Portanto, o objetivo deste trabalho é avaliar retrospectivamente as histerectomias realizadas na Disciplina de Ginecologia e Obstetrícia da Faculdade de Medicina do Triângulo Mineiro.

\section{MÉTODO}

Realizou-se um estudo retrospectivo de 554 pacientes submetidas à histerectomia selecionadas no período de 1988 a 1998 no Hospital Escola da FMTM, que é um centro regional de referência terciário e atende pelo Sistema Único de Saúde. Deste total, $470(84,8 \%)$ foram abdominais e 84 $(15,2 \%)$ vaginais.

A faixa etária das pacientes submetidas à operação por via abdominal e vaginal foi, respectivamente, menos de 30 anos: $11(2,3 \%)$ e $4(4,8 \%), 31$ a 40 anos: $132(28,1 \%)$ e 8 $(9,5 \%), 41$ a 50 anos: $196(41,7 \%)$ e $20(23,8 \%), 51$ a 60 anos $80(17,1 \%)$ e $17(20,2 \%)$ e mais que 60 anos: $51(10,8 \%)$ e 35 $(41,7 \%)$. A média de idade nos procedimentos abdominais foi de 45,1 \pm 9,5 anos e nos vaginais de 57,1 \pm 14 anos. Os dados coletados foram indicações, complicações, antibioticoterapia, técnica operatória, número de transfusões sangüíneas e tipo de incisão. O tempo cirúrgico, a duração da internação e as complicações foram separados de acordo com a incisão abdominal em vertical e horizontal. Na análise estatística, utilizaram-se os testes exatos de Fisher e Quiquadrado $\left(\mathrm{X}^{2}\right)$ com nível de significância menor que 0,05 .

\section{RESULTADOS}

A miomatose uterina foi a indicação mais comum para a via abdominal (ver Tabela 1). As outras indicações relatadas nesta tabela foram, mais freqüentemente, neoplasias malignas do ovário e útero, doença trofoblástica gestacional, pólipos endometriais, endometriose e duas ou mais alterações associadas. As complicações intra-operatórias aconte- ceram em $1,3 \%$ do total de 470 operações. Os tipos de intercorrências estão na Tabela 2 . A Tabela 3 mostra os problemas pós-operatórios, que corresponderam a $2,4 \%$ dos procedimentos realizados. Observa-se que não houve diferença estatística entre os números de cada complicação intra-operatória quando se compara a incisão vertical com a transversal. Na Tabela 4, está o tempo cirúrgico. Verifica-se que este foi estatisticamente maior nas incisões longitudinais. A transfusão sangüínea no intra e/ou no pós-operatório foi realizada em $31(6,6 \%)$ pacientes. Na Tabela 5 mostra-se o período da internação hospitalar, estatisticamente maior nas pacientes que se submeteram à histerectomia por incisão vertical. A antibioticoterapia profilática foi utilizada em $453(96,4 \%)$ casos, sendo os derivados da penicilina em 391 (86,3\%) usados em média sete dias com dose padrão. Na Tabela 6 estão as indicações das ressecções vaginais. A duração deste procedimento foi em torno de quatro horas em $36(42,8 \%)$ casos e de três horas em 34 (40,5\%). Em três oportunidades (3,6\%), houve necessidade de transfusão sangüínea. $O$ tempo de internação foi de três a cinco dias em $58(69,0 \%)$ descritos. O antibiótico profilático mais utilizado foi o derivado da penicilina em $54(64,3 \%)$ pacientes.

Tabela 1

Indicações das histerectomias abdominais

\begin{tabular}{lrc}
\hline & $N^{o}$ & $\%$ \\
\hline Miomatose uterina & 288 & 61,3 \\
Neoplasia maligna do colo do útero & 69 & 14,7 \\
Neoplasia benigna do ovário & 20 & 4,2 \\
Mioma e cisto de ovário & 13 & 2,8 \\
Hemorragia disfuncional uterina & 8 & 1,7 \\
Hiperplasia endometrial atípica & 7 & 1,5 \\
Outras & 65 & 13,8 \\
\hline Total & 470 & 100,0 \\
\hline
\end{tabular}

Tabela 2

Complicações intra-operatórias das histerectomias abdominais

\begin{tabular}{|c|c|c|c|c|c|c|}
\hline \multirow[b]{2}{*}{ Complicação** } & \multicolumn{2}{|c|}{ Vertical $^{*}$} & \multicolumn{2}{|c|}{ Transversa } & \multicolumn{2}{|c|}{ Total } \\
\hline & $N^{o}$ & $\%$ & $N^{o}$ & $\%$ & $N^{o}$ & $\%$ \\
\hline Lesão ureteral & 2 & 18,2 & 1 & 20,0 & 3 & 18,8 \\
\hline Lesão retal & 1 & 9,1 & - & - & 1 & 6,3 \\
\hline Lesão vesical & - & - & 1 & 20,0 & 1 & 6,3 \\
\hline Lesão de intestino delgado & 1 & 9,1 & - & - & 1 & 6,3 \\
\hline Hemorragia intra-operatória & 7 & 63,6 & 3 & 60,0 & 10 & 62,3 \\
\hline Total & 11 & 100 & 5 & 100 & 16 & 100 \\
\hline
\end{tabular}

*P: não significante, teste exato de Fisher. Comparação entre o número de complicações da incisões transversa versus vertical; $* * P$ : não significante, teste de $\chi 2$. Comparação entre o número de cada complicação das incisões transversa versus vertical. 
Tabela 3

Complicações pós-operatórias das histerectomias abdominais

\begin{tabular}{|c|c|c|c|c|c|c|}
\hline \multirow[b]{2}{*}{ Complicações** } & \multicolumn{2}{|c|}{ Vertical* } & \multicolumn{2}{|c|}{ Transversa } & \multicolumn{2}{|c|}{ Total } \\
\hline & $N^{\underline{o}}$ & $\%$ & $N^{o}$ & $\%$ & $N^{\underline{o}}$ & $\%$ \\
\hline Abscesso ou hematoma de cicatriz & 3 & 23,0 & 3 & 75,0 & 6 & 35,3 \\
\hline Hérnia incisional & 4 & 30,8 & 1 & 25,0 & 5 & 29,4 \\
\hline Evisceração & 4 & 30,8 & - & - & 4 & 23,5 \\
\hline Abdome agudo obstrutivo & 1 & 7,7 & - & - & 1 & 5,9 \\
\hline Trombose venosa profunda & 1 & 7,7 & - & - & 1 & 5,9 \\
\hline Total & 13 & 100 & 4 & 100 & 17 & 100 \\
\hline
\end{tabular}

$* P<0,05$, teste exato de Fisher. Comparação entre o número de complicações da incisões transversa versus vertical;

**P: não significante, teste de $\chi 2$. Comparação entre o número de cada complicação das incisões transversa versus vertical.

Tabela 4

Tempo cirúrgico em horas das histerectomias abdominais

\begin{tabular}{|c|c|c|c|c|c|c|}
\hline \multirow[b]{2}{*}{ Tempo Cirúrgico (horas) } & \multicolumn{2}{|c|}{ Vertical* } & \multicolumn{2}{|c|}{ Transversa } & \multicolumn{2}{|c|}{ Total } \\
\hline & $N^{o}$ & $\%$ & $N^{o}$ & $\%$ & $N^{\underline{o}}$ & $\%$ \\
\hline 2 & 4 & 1,5 & 8 & 3,7 & 12 & 2,6 \\
\hline 3 & 42 & 16,6 & 60 & 27,6 & 102 & 21,7 \\
\hline 4 & 111 & 43,9 & 91 & 42 & 202 & 43,0 \\
\hline 5 & 51 & 20,2 & 45 & 20,7 & 96 & 20,4 \\
\hline$>6$ & 45 & 17,8 & 13 & 6,0 & 58 & 12,3 \\
\hline Total & 253 & 100 & 217 & 100 & 470 & 100 \\
\hline
\end{tabular}

$* P<0,001$, teste do $\chi 2$. Comparação entre incisão transversa versus vertical.

Tabela 5

Tempo de internação em dias das pacientes submetidas à histerectomia abdominal

\begin{tabular}{|c|c|c|c|c|c|c|}
\hline \multirow[b]{2}{*}{ Tempo de internação (dias) } & \multicolumn{2}{|c|}{ Vertical* } & \multicolumn{2}{|c|}{ Transversa } & \multicolumn{2}{|c|}{ Total } \\
\hline & $N^{o}$ & $\%$ & $N^{o}$ & $\%$ & $N^{o}$ & $\%$ \\
\hline 3 & 27 & 10,7 & 38 & 17,5 & 65 & 13,8 \\
\hline 4 & 81 & 32,0 & 101 & 46,5 & 182 & 38,7 \\
\hline 5 & 78 & 30,8 & 41 & 18,9 & 119 & 25,3 \\
\hline 6 & 19 & 7,5 & 16 & 7,4 & 35 & 7,5 \\
\hline$>7$ & 48 & 19,0 & 21 & 9,7 & 69 & 14,7 \\
\hline Total & 253 & 100 & 217 & 100 & 470 & 100 \\
\hline
\end{tabular}

${ }^{*} P<0,001$, teste do $\chi 2$. Comparação entre incisão transversa versus vertical.

Tabela 6

Indicações de histerectomias vaginais

\begin{tabular}{l|c|c}
\hline Indicações & $N^{o}$ & $\%$ \\
\hline Prolapso de I grau & 25 & 29,8 \\
Prolapso de II grau & 48 & 57,1 \\
Prolapso de III grau & 9 & 10,7 \\
Prolapso de III e câncer de colo uterino & 2 & 2,4 \\
\hline Total & 84 & 100 \\
\hline
\end{tabular}

\section{DISCUSSÃO}

A indicação mais freqüente de histerectomia por alteração benigna nos serviços de ginecologia é o mioma uterino. A freqüêencia varia em média de $70 \%$ a $80 \%$ dos $\operatorname{casos}^{2,5,6}$. Neste trabalho, foi a principal indicação. As neoplasias malignas de colo do útero são a segunda causa das indicações do procedimento. Isto se deve ao fato de sermos também centro de referência em oncologia ginecológica.

O tipo de incisão abdominal mais empregado foi o vertical. A indicação do acesso a ser realizado no serviço 
não obedece a um padrão rígido. Pacientes não obesas, com útero pequeno e/ou até a altura da cicatriz umbilical e sem intervenção abdominal anterior têm a preferência pela abertura transversa tipo Pfannenstiel, nos casos oncológicos, principalmente provenientes do colo uterino, a de Cherney. A justificativa é um melhor resultado estético no pós-operatório, sem prejudicar uma boa exposição da pelve durante a operação ${ }^{7}$.

A histerectomia está associada a uma série de complicações. No entanto, a maioria é de pequena morbidez e o risco de morte é baixo ${ }^{2}$. A lesão do trato urinário, embora séria, é infreqüente. A ureteral acontece de 0,2 a $0,5 \%$ das cirurgias e a vesical de 0,3 a $0,8 \%{ }^{8-12}$. Nossos resultados demonstraram incidência semelhante. As injúrias do ureter e bexiga durante a histerectomia devem ser tratadas imediatamente no intra-operatório, pois requerem técnicas cirúrgicas mais simples e evitam maior trauma para a paciente ${ }^{13}$.

As complicações pós-operatórias aconteceram em cerca de 3,6\% dos casos. Esta percentagem é semelhante à descrita na literatura ${ }^{2,5}$. Observamos que estas alterações intra e pós-operatórias foram mais freqüentes nas incisões longitudinais, sendo estatisticamente significante no pósoperatório. Entretanto, como já descrito anteriormente, existe uma indicação de qual incisão deve ser utilizada. Isto também se reflete no tempo cirúrgico e no período de internação, os quais foram estatisticamente maiores nos acessos longitudinais.

O excesso de sangramento durante a histerectomia, que levou à necessidade de transfusão sangüínea, aconteceu em $6,6 \%$ dos casos. Na literatura, a média deste procedimento é em torno de $15 \%$. Esta percentagem é o dobro da encontrada neste trabalho, entretanto pode ser explicada pela necessidade de reposição volêmica por anemia préexistente ${ }^{14,15}$.

As cefalosporinas de primeira geração foram a as drogas mais utilizadas para antibioticoterapia profilática. Devido ao baixo índice de infecção encontrado neste estudo, entendemos que ela é a droga de escolha para uso pré-operatório nas histerectomias não complicadas. As vantagens das mesmas recaem sobre a sua eficácia e custo mais barato ${ }^{16}$.

A indicação mais freqüente de histerectomia vaginal neste trabalho foi o prolapso uterino associado ou não a outras alterações. Dados da literatura relatam taxas que variam de 31 a $39 \%$ para este tipo de problema ${ }^{14,17}$. A percentagem maior ocorre principalmente na América do Norte, devido a outros fatores não comuns no Brasil, como a esterilização ${ }^{18,19}$. Em um estudo realizado por Amirikia \& Evans ${ }^{5}$, de um total de 6.435 ressecções uterinas, $33 \%$ foram histerectomia vaginal. Nossos resultados demonstraram uma freqüência menor da exérese vaginal em relação à ablação abdominal. Isto é explicado porque a extirpação do útero em nosso serviço está relacionada com a presença do prolapso. Em dois casos, havia o diagnóstico de câncer de colo uterino e a operação realizada foi a técnica descrita por Schauta ${ }^{20}$.

Concluímos que as histerectomias abdominais e vaginais, quando bem indicadas, contribuem para melhorar a qualidade de vida das mulheres. Procedimentos técnicos, como a incisão a ser utilizada, irão depender do biótipo da paciente e da doença. O risco de morbidez para a paciente é baixo, não obstante a procura de resultados cada vez melhores justifique a realização de revisões críticas freqüentes e atualizadas das suas indicações e contra-indicações.

\begin{abstract}
Objective: Hysterectomy is a frequently performed surgery, but national literature gives limited information regarding indications, technique and complications. The aim of this study is to evaluate our results with this operation performed by the Discipline of Gynecology and Obstetrics of the Faculty of Medicine of "Triângulo Mineiro". Methods: A retrospective study of 470 abdominal and 84 vaginal hysterectomies was conducted in which the indications, operation time, abdominal approach, hospitalization time, and morbidity were analyzed. Results: Uterine myoma and uterine prolapse were the most frequent indications for abdominal and vaginal hysterectomies, respectively. Intra-operative (3.4\%) and postoperative (2.4\%) complications occurred in abdominal hysterectomies, with no significant statistical difference in the incidence of complications related to the type of incision (transverse or vertical). Operative time and hospital stay were significantly increased with the vertical incision. Hemorrhage was the major intraoperative complication and wound infection in postoperative complications. Conclusions: We concluded that hysterectomy is a low risk procedure although improvements in surgical technique and continued research are needed for a even safer procedure.
\end{abstract}

Key Words: Abdominal hysterectomy; Vaginal hysterectomy; Indication; Complication; Abdominal incision. 


\section{REFERÊNCIAS}

1. Benrubi GI. History of hysterectomy. Special article Florida MA, 1988, 75(8):533-538.

2. Easterday LC, Grimes DA, Riggs JA. Hysterectomy in the United States. Obstet Gynecol, 1993, 62:203-212.

3. Thompsom JD, Birch HW: Indications for hysterectomy. Clin Obstetric Gynecol, 1981, 24(04):1245-1258.

4. Steege JF. Indications for hysterectomy: Have they changed? Clin Obstet Gynecol, 1997, 40(4):878-885.

5. Amirikia H, Evans TN. The-year review of hysterectomies: trends, indications and risks. Am J Obstet Gynecol, 1979, 134:431-437.

6. Simões PM. Histerectomias abdominais. Femina, 1986, 14(06):524-533.

7. Baker VV, Shingleton HM. Incisions. Clin Obstet Gynecol 1988; 31(3):700-711.

8. White SC, Wartel LJ, Wade ME. Comparison of abdominal and vaginal hysterectomies. A review of 600 operations. Obstet Gynecol, 1971, 37:530-537.

9. Everett HS, Mattingly RF. Urinary tract injuries resulting from pelvic surgery. Am J Obstet Gynecol, 1956, 71:502-504.

10. Thompson JD, Benigno BB. Vaginal repair of ureteral injuries. Am J Obstet Gynecol, 1971, 111: 601-604.

11. Stumpf PG, Ballard CA, Lowensohn R. Abdominal hysterectomy for abortion-sterilization. A report 500 consecutive cases. Am J Obstet Gynecol, 1980, 136:714720.

12. Harris WJ. Complications of hysterectomy. Clin Obstet Gynecol, 1997, 40(4): 928-937.

13. Kostakopoulus A, Deliveliotiis C, Louras G et al. Early repair of injury to the ureter or bladder after hysterectomy. Int Urol Nephrol, 1998, 30(04):445-450.
14. Dicker RC, Greenspan JR, Strauss LT et al. Complications of abdominal and vaginal hysterectomies among women of reproductive age in the United States. Am J Obstet Gynecol, 1982, 144:841-848.

15. Ledger WJ, Child MA: The hospital care of patients undergoing hysterectomy: $\mathrm{Na}$ analysis of 12,026 patients from the Professional Activity Study. Am J Obstet Gynecol, 1973, 117:423-433.

16. Capen CV. Gynecologic Surgery: Preoperative evaluation. Clin Obstet Gynecol, 1988, 31(3): 673-685.

17. Copenhaver EH . Vaginal hysterectomy. An analysis of indications and complications among 1.000 operations. Am J Obstet Gynecol, 1962, 84:123-126.

18. Van Nagel JR Jr, Roddick JW. Vaginal hysterectomy as sterilization procedure. Am J Obstet Gynecol, 1971, 111:703-705.

19. Atkinson SM Jr, Chappell SM. Vaginal hysterectomy for sterilization. Obstet Gynecol, 1972, 39:759-766.

20. Salvatore CA. Histerectomia vaginal radical. In: Salvatore CA (ed). Ginecologia Operatória. $1^{\mathrm{a}}$ ed. Rio de Janeiro, Guanabara Koogan: 124-133, 1974.

Endereço para correspondência:

Dr. Eddie Fernando Cândido Murta

Faculdade de Medicina do Triângulo Mineiro

Disciplina de Ginecologia e Obstetrícia

Av. Getúlio Guaritá, S/N

38025-440 - Uberaba-MG

E-mail: eddiemurta@mednet.com.br 